\title{
SEARCH FOR ANISOTROPY OF ULTRAHIGH ENERGY COSMIC RAYS WITH THE TELESCOPE ARRAY EXPERIMENT
}

\begin{abstract}
T. Abu-Zayyad ${ }^{1}$, R. Aida ${ }^{2}$, M. Allen ${ }^{1}$, R. Anderson ${ }^{1}$, R. Azuma ${ }^{3}$, E. Barcikowski ${ }^{1}$, J. W. Belz ${ }^{1}$, D. R. Bergman ${ }^{1}$,

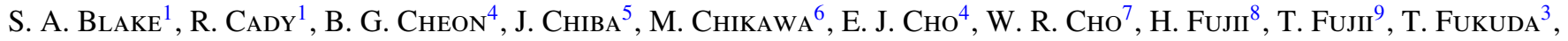

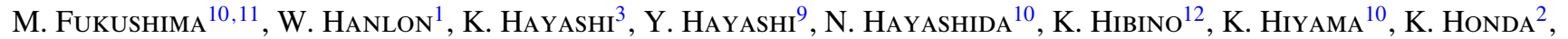

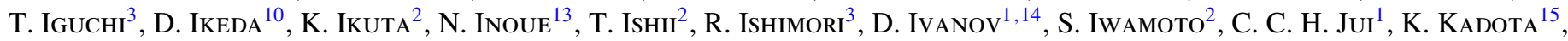
F. KaKimoto ${ }^{3}$, O. Kalashev ${ }^{16}$, T. Kanbe $^{2}$, K. Kasahara ${ }^{17}$, H. Kawai ${ }^{18}$, S. KawaKami ${ }^{9}$, S. Kawana ${ }^{13}$, E. Kido ${ }^{10}$, H. B. Kim ${ }^{4}$, H. K. Kim ${ }^{7}$, J. H. Kim ${ }^{4}$, J. H. Kim ${ }^{19}$, K. Kitamoto ${ }^{6}$, S. Kitamura ${ }^{3}$, Y. Kitamura ${ }^{3}$, K. KobaYAshi ${ }^{5}$, Y. Kobayashi ${ }^{3}$, Y. Kondo ${ }^{10}$, K. Kuramoto ${ }^{9}$, V. KuZmin ${ }^{16}$, Y. J. Kwon ${ }^{7}$, S. I. Lim $^{20}$, S. Machida ${ }^{3}$, K. Martens ${ }^{11}$, J. Martineau ${ }^{1}$, T. Matsuda ${ }^{8}$, T. Matsuura ${ }^{3}$, T. Matsuyama ${ }^{9}$, J. N. Matthews ${ }^{1}$, M. Minamino $^{9}$, K. Miyata ${ }^{5}$, Y. Murano ${ }^{3}$, I. Myers $^{1}$, K. Nagasawa $^{13}$,

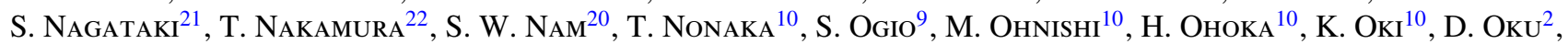
T. OKuda ${ }^{23}$, A. Oshima ${ }^{9}$, S. Ozawa ${ }^{17}$, I. H. Park ${ }^{20}$, M. S. Pshirkov ${ }^{24}$, D. C. RodrigueZ ${ }^{1}$, S. Y. Roh ${ }^{19}$, G. RubTsOv ${ }^{16}$, D. Ryu ${ }^{19}$, H. Sagawa ${ }^{10}$, N. Sakurai ${ }^{9}$, A. L. Sampson ${ }^{1}$, L. M. Scott ${ }^{14}$, P. D. Shah ${ }^{1}$, F. Shibata ${ }^{2}$, T. Shibata ${ }^{10}$, H. Shimodaira ${ }^{10}$, B. K. Shin ${ }^{4}$, J. I. Shin ${ }^{7}$, T. Shirahama ${ }^{13}$, J. D. Smith ${ }^{1}$, P. Sokolsky ${ }^{1}$, T. J. Sonley ${ }^{1}$, R. W. SPRinger ${ }^{1}$, B. T. Stokes ${ }^{1}$,

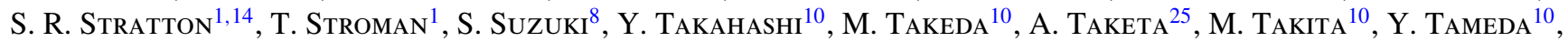
H. Tanaka ${ }^{9}$, K. Tanaka ${ }^{26}$, M. Tanaka ${ }^{9}$, S. B. Thomas ${ }^{1}$, G. B. Thomson ${ }^{1}$, P. Tinyakov ${ }^{16,24}$, I. Tkachev ${ }^{16}$, H. Tokuno ${ }^{3}$,

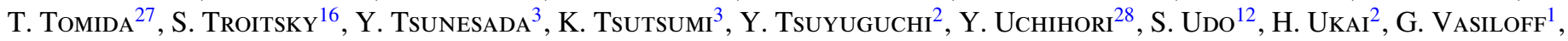

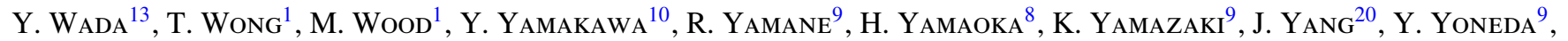

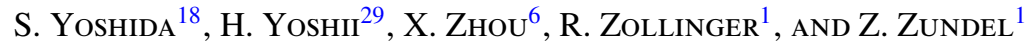

${ }^{1}$ High Energy Astrophysics Institute and Department of Physics and Astronomy, University of Utah, Salt Lake City, UT, USA

${ }^{2}$ Interdisciplinary Graduate School of Medicine and Engineering, University of Yamanashi, Kofu, Yamanashi, Japan

${ }^{3}$ Graduate School of Science and Engineering, Tokyo Institute of Technology, Meguro, Tokyo, Japan

${ }^{4}$ Department of Physics and The Research Institute of Natural Science, Hanyang University, Seongdong-gu, Seoul, Republic of Korea

${ }^{5}$ Department of Physics, Tokyo University of Science, Noda, Chiba, Japan

${ }^{6}$ Department of Physics, Kinki University, Higashi Osaka, Osaka, Japan

${ }^{7}$ Department of Physics, Yonsei University, Seodaemun-gu, Seoul, Republic of Korea

${ }^{8}$ Institute of Particle and Nuclear Studies, KEK, Tsukuba, Ibaraki, Japan

${ }^{9}$ Graduate School of Science, Osaka City University, Osaka, Osaka, Japan

${ }^{10}$ Institute for Cosmic Ray Research, University of Tokyo, Kashiwa, Chiba, Japan

${ }^{11}$ Kavli Institute for the Physics and Mathematics of the Universe, University of Tokyo, Kashiwa, Chiba, Japan

${ }^{12}$ Faculty of Engineering, Kanagawa University, Yokohama, Kanagawa, Japan

${ }^{13}$ The Graduate School of Science and Engineering, Saitama University, Saitama, Saitama, Japan

${ }^{14}$ Department of Physics and Astronomy, Rutgers University, Piscataway, USA

${ }^{15}$ Department of Physics, Tokyo City University, Setagaya-ku, Tokyo, Japan

${ }^{16}$ Institute for Nuclear Research of the Russian Academy of Sciences, Moscow, Russia

${ }^{17}$ Advanced Research Institute for Science and Engineering, Waseda University, Shinjuku-ku, Tokyo, Japan

${ }^{18}$ Department of Physics, Chiba University, Chiba, Chiba, Japan

${ }^{19}$ Department of Astronomy and Space Science, Chungnam National University, Yuseong-gu, Daejeon, Republic of Korea

${ }^{20}$ Department of Physics and Institute for the Early Universe, Ewha Womans University, Seodaaemun-gu, Seoul, Republic of Korea

${ }^{21}$ Yukawa Institute for Theoretical Physics, Kyoto University, Sakyo, Kyoto, Japan

${ }^{22}$ Faculty of Science, Kochi University, Kochi, Kochi, Japan

${ }^{23}$ Department of Physical Sciences, Ritsumeikan University, Kusatsu, Shiga, Japan

${ }^{24}$ Department of Physical Theorique, University Libre de Bruxelles, Brussels, Belgium

${ }^{25}$ Earthquake Research Institute, University of Tokyo, Bunkyo-ku, Tokyo, Japan

${ }^{26}$ Department of Physics, Hiroshima City University, Hiroshima, Hiroshima, Japan

${ }^{27}$ RIKEN, Advanced Science Institute, Wako, Saitama, Japan

${ }^{28}$ National Institute of Radiological Science, Chiba, Chiba, Japan

${ }^{29}$ Department of Physics, Ehime University, Matsuyama, Ehime, Japan

Received 2012 May 27; accepted 2012 July 20; published 2012 August 31

\begin{abstract}
We study the anisotropy of Ultra-High Energy Cosmic Ray (UHECR) events collected by the Telescope Array (TA) detector in the first 40 months of operation. Following earlier studies, we examine event sets with energy thresholds of $10 \mathrm{EeV}, 40 \mathrm{EeV}$, and $57 \mathrm{EeV}$. We find that the distributions of the events in right ascension and declination are compatible with an isotropic distribution in all three sets. We then compare with previously reported clustering of the UHECR events at small angular scales. No significant clustering is found in the TA data. We then check the events with $E>57 \mathrm{EeV}$ for correlations with nearby active galactic nuclei. No significant correlation is found. Finally, we examine all three sets for correlations with the large-scale structure (LSS) of the universe. We find that the two higher-energy sets are compatible with both an isotropic distribution and the hypothesis that UHECR sources follow the matter distribution of the universe (the LSS hypothesis), while the event set with $E>10 \mathrm{EeV}$ is compatible with isotropy and is not compatible with the LSS hypothesis at 95\% CL unless large deflection angles
\end{abstract}


are also assumed. We show that accounting for UHECR deflections in a realistic model of the Galactic magnetic field can make this set compatible with the LSS hypothesis.

Key words: acceleration of particles - astroparticle physics - cosmic rays - magnetic fields - methods: statistical - relativistic processes

Online-only material: color figures

\section{INTRODUCTION}

One of the keys to understanding the nature of the Ultra-High Energy Cosmic Rays (UHECRs) is their distribution over the sky. This distribution depends on the UHECR sources, as well on the UHECR mass composition and large-scale magnetic fields, both Galactic and extragalactic. Despite significant effort, none of these issues is presently well understood.

Observation of the cutoff in the highest-energy part of the cosmic-ray spectrum (Abbasi et al. 2008a; Abraham et al. 2008b) suggests that the UHECR propagation at high energies is limited by the interaction with the cosmic background radiation (the Greisen-Zatsepin-Kuzmin (GZK) effect (Greisen 1966; Zatsepin \& Kuzmin 1966)). One therefore expects that the closest sources of UHECRs are situated within the GZK volume of the size $\lesssim 100 \mathrm{Mpc}$. At these scales the matter distribution in the universe is inhomogeneous, and so must be the distribution of the UHECR sources. If propagation of UHECRs at these distances is quasi-rectilinear (whether or not this is the case depends on both their composition and the magnetic fields), one generally expects the UHECR flux to be anisotropic, showing variations at large angular scales and possibly point sources.

If UHECR primary particles are protons, as suggested by the composition measurements performed by the High Resolution Fly's Eye (HiRes) and the Telescope Array (TA) experiments (Abbasi et al. 2010b; Tameda 2010), the UHECR propagation is, in fact, expected to be quasi-rectilinear. With the existing estimates of the Galactic magnetic field (GMF; Han et al. 2006; Sun et al. 2008; Pshirkov et al. 2011) and bounds on the extragalactic ones (Kronberg 1994), the deflections of protons should be relatively small. For instance, a random extragalactic field of magnitude $1 \mathrm{nG}$ and correlation length of $\sim 1 \mathrm{Mpc}$ would deflect a proton of energy $10^{20} \mathrm{eV}$ by about $2^{\circ}$ over a distance of $50 \mathrm{Mpc}$, while the Galactic field would produce deflections of the order of $2^{\circ}-4^{\circ}$ depending on the direction. In this case, a sizeable anisotropy may be expected regardless of the density of the UHECR sources down to energies as low as $10^{19} \mathrm{eV}$.

On the contrary, if the composition at highest energies is heavy or predominantly heavy, as the results of the Pierre Auger Observatory (PAO; Abraham et al. 2010) seem to indicate, the quasi-rectilinear propagation is not expected for the bulk of UHECRs. Some anisotropy at large angles may still arise if the extragalactic fields are sufficiently small and the density of sources is such that only a few nearby ones contribute to the observed flux, but the small-scale anisotropy would be suppressed (for recent analyses see, e.g., Giacinti et al. 2010; Takami et al. 2012). Thus, the study of the UHECR anisotropy may shed light on both the mass composition and the density of the UHECR sources (Dubovsky et al. 2000; Yoshiguchi et al. 2003, 2004; Kachelriess \& Semikoz 2005).

Numerous attempts at detection of the UHECR anisotropy have been made previously. Early studies indicated clustering of the UHECR events at small angular scales (Hayashida et al. 1996; Tinyakov \& Tkachev 2001). On the basis of small-scale correlations, different classes of putative sources of UHECR were suggested (see, e.g., Gorbunov et al. 2004; Abbasi et al. 2006; Abraham et al. 2007, 2008a). More recently, the PAO has claimed correlations of UHECRs with the nearby active galactic nuclei (AGNs; Abraham et al. 2007, 2008a) which were not confirmed by observations in the Northern hemisphere (Abbasi et al. 2008b).

At larger angular scales, evidence for correlations with the supergalactic plane was claimed by Stanev et al. (1995), Glushkov (2001), and Glushkov \& Pravdin (2001) but not confirmed by other authors (Hayashida et al. 1996; Kewley et al. 1996; Bird et al. 1999). Also, Kashti \& Waxman (2008) found the anisotropy in the PAO data which was not confirmed by the HiRes data in the Northern hemisphere (Abbasi et al. 2010a).

In this paper, we present the anisotropy analysis of UHECR observed by the surface detector (SD) of the TA in the first 40 months of its operation. TA is a hybrid UHECR detector located in the Northern hemisphere in Utah, USA $\left(39^{\circ} 17^{\prime} 48^{\prime \prime} \mathrm{N}\right.$, $\left.112^{\circ} 54^{\prime} 31^{\prime \prime} \mathrm{W}\right)$ which has been fully operational since 2008 March. It consists of 507 scintillator detectors covering the area of approximately $700 \mathrm{~km}^{2}$ (for details see Abu-Zayyad et al. 2012b). The atmosphere over the surface array is viewed by 38 fluorescence telescopes arranged in three stations (see Tokuno et al. 2012). The SD of TA is the largest in the Northern hemisphere.

In this paper, we focus on testing previous observations. Namely, we consider the clustering of the UHECR events at small angular scales (as would be produced by bright point sources), possible correlation with nearby AGNs, and correlation of the TA events with the large-scale structure (LSS) of the universe. Following previous analyses, we consider three a priori chosen energy thresholds: $10 \mathrm{EeV}, 40 \mathrm{EeV}$, and $57 \mathrm{EeV}$. It should be noted that different experiments may have different energy scales due to different systematic errors in the energy determination, which may affect the selection of the events. When referring to the results of other experiments, we assume the energy scales as reported by these experiments. In statistical tests which require a pre-defined confidence level we set the latter to $95 \%$.

The paper is organized as follows. In Section 2, we describe the data sets used. In Section 3, we examine the event sets for a presence of small-scale clustering by studying the UHECR autocorrelation function. In Section 4, we consider correlations of UHECR events with nearby AGNs. Section 5 describes our search for correlations of the UHECR events with the LSS of the universe. In Section 6, we summarize the results and present conclusions.

\section{DATA}

Among the existing TA data sets (SD data, Fluorescence Detector (FD) data in mono and stereo modes, and hybrid detector data) the SD data set has by far the largest number of events. The present analysis is based on the data collected in the period 2008 May 11-2011 September 15 (40 months) 

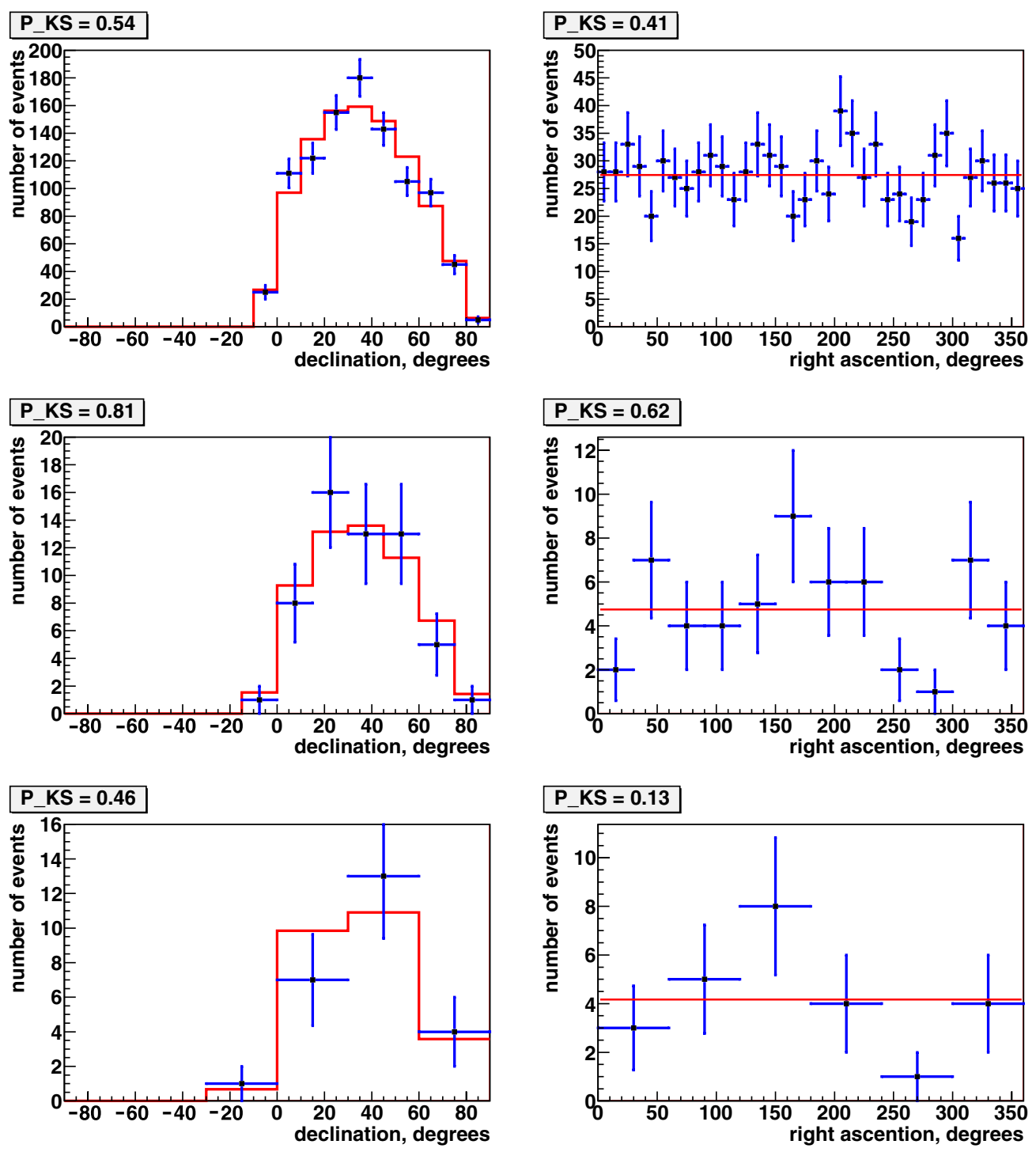

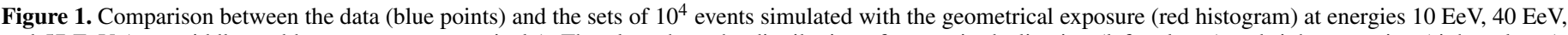

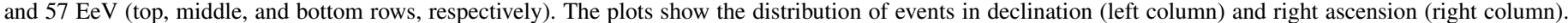
The compatibility of the two distributions by the Kolmogorov-Smirnov test is given as $P_{\mathrm{KS}}$ in the upper left corner of each plot.

(A color version of this figure is available in the online journal.)

of operation by the TA SD array. Cutting events with zenith angle $>45^{\circ}$, the SD data set contains 988 events with energies $>10 \mathrm{EeV}, 57$ events with $E>40 \mathrm{EeV}$, and 25 events with $E>57 \mathrm{EeV}$. This is the largest UHECR set to date in the Northern hemisphere.

The angular resolution of TA events with $E>10 \mathrm{EeV}$ is approximately 1.5 . This follows from the comparison of the thrown and reconstructed arrival directions of simulated data sets and is supported by the direct comparison between the SD and FD arrival directions of hybrid events. The energy resolution of the TA SD at $E>10 \mathrm{EeV}$ is better than $20 \%$ (Abu-Zayyad et al. 2012a).

The exposure of the TA surface array is calculated by the Monte Carlo (MC) technique with full simulation of the detector, which will be described elsewhere. As follows from the MC simulations, the acceptance of the TA SD for $E>10 \mathrm{EeV}$ and zenith angle cut of $45^{\circ}$ is close to the geometrical one. For reasons of computational efficiency, in the present analysis aimed at anisotropy at relatively small angular scales we use the geometrical acceptance to generate random event sets.
Figure 1 shows the comparison between the distributions in declination (left column) and right ascension (right column) of the events simulated with the geometrical exposure (red line) and the data (blue data points) at the energy thresholds of $10 \mathrm{EeV}$, $40 \mathrm{EeV}$, and $57 \mathrm{EeV}$ (top, middle, and bottom rows, respectively). The compatibility of expected and observed distributions in all six cases was checked by the Kolmogorov-Smirnov (K-S) test. The lowest K-S probability was $p=0.13$ for the distribution in the right ascension at $E>57 \mathrm{EeV}$. Thus, all three sets are compatible with a uniform distribution.

\section{AUTOCORRELATION FUNCTION}

The AGASA experiment reported clustering of UHECR events with $E>40 \mathrm{EeV}$ at the angular scale of 2.5 (Hayashida et al. 1996). Here, we repeat this analysis using the TA data set.

The procedure is as follows: for a given angular separation, $\delta$, we count the number of pairs of observed events that are separated by an angular distance less than $\delta$, thus obtaining the data count. We then generate a large number (typically, $10^{5}$ ) of 

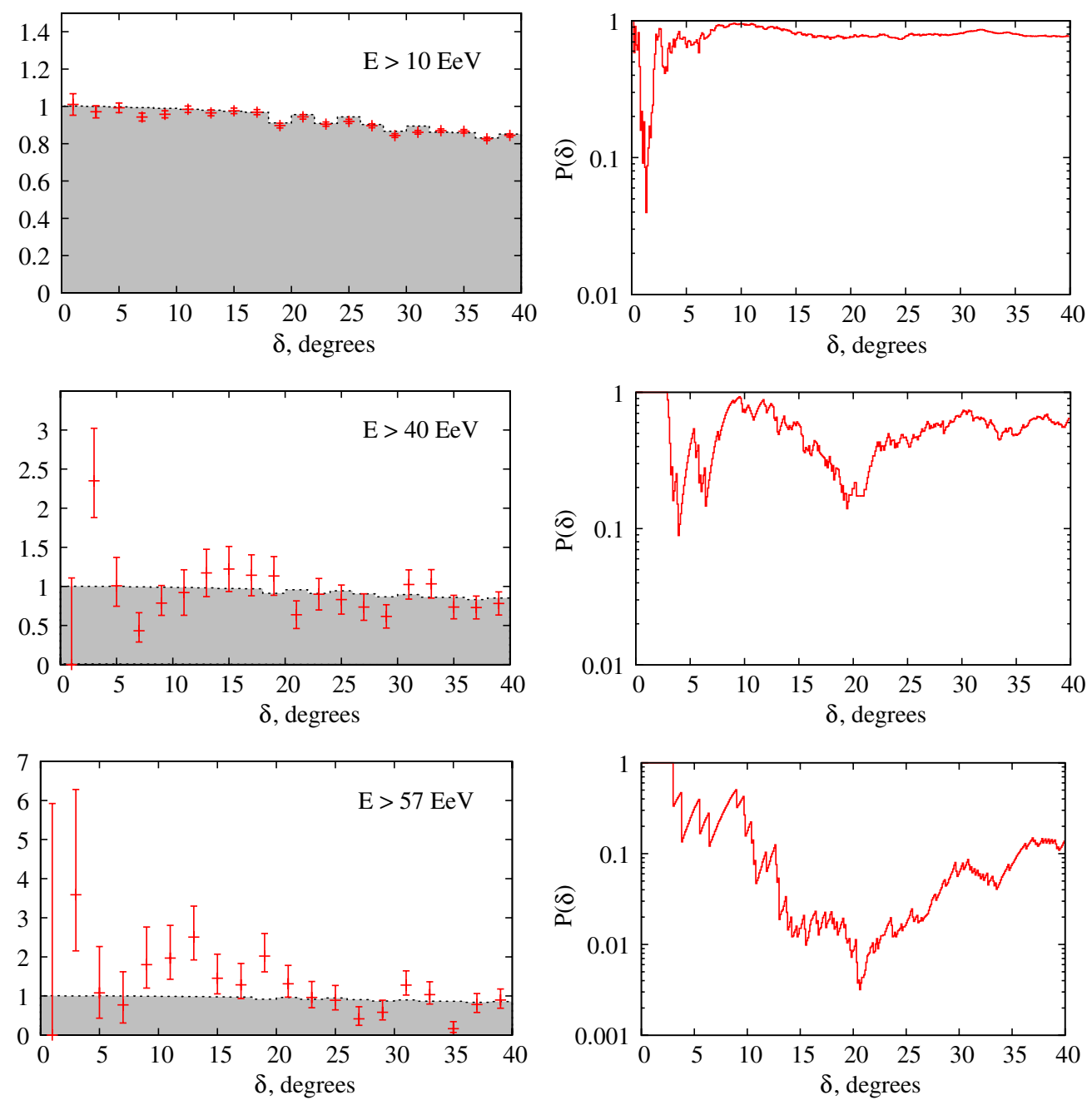

Figure 2. Autocorrelations in the TA data sets at $E>10 \mathrm{EeV}, E>40 \mathrm{EeV}$, and $E>57 \mathrm{EeV}$ (top, middle, and bottom rows, respectively). Left panels: the number of pairs with angular separations $\delta$ normalized to the area of the angular bin (data points), compared to the expectation for the uniform distribution (shaded histogram). The errors are $1 \sigma$ Poisson errors. Right panels: probability, $P(\delta)$, that the excess of pairs with the angular separation less than $\delta$ occurs as a fluctuation in a uniform distribution. Small $P(\delta)$ indicates a departure from isotropy.

(A color version of this figure is available in the online journal.)

MC event sets each having the same number of events as the real data set. The simulated sets are generated with a uniform distribution according to the TA exposure. In each MC set we count pairs of events in the same way as in the data, which gives the MC count for that set. We then calculate the average MC count for all of the MC sets. This represents the expected number of pairs for the angular scale $\delta$, assuming a uniform cosmic-ray distribution. For each value of $\delta$ we then determine the fraction of simulated sets where the number of pairs is greater than or equal to the number of pairs in the data. This gives the $p$-value, $P(\delta)$, which describes how likely the excess of pairs, if found in the data, is to occur as a result of a fluctuation in a random set. Small values of $P(\delta)$ thus indicate a departure from uniformity at the corresponding angular scale.

We first perform a blind test of the AGASA claim. Fixing the energy threshold to $40 \mathrm{EeV}$ and the separation angle to $\delta=2.5$ we find 0 pairs while 1.5 pairs are expected in the case of a uniform distribution. Therefore, there is no excess of small-scale clusters in the TA data.

We next extend the analysis to all angular scales. No significant excess is found. The results are illustrated in Figure 2 for angles from $0^{\circ}$ to $40^{\circ}$ and three energy thresholds of $10 \mathrm{EeV}$,
$40 \mathrm{EeV}$, and $57 \mathrm{EeV}$ as specified on the plots. For each energy threshold, the left panel shows the number of pairs with the angular separations $\delta$ binned in $2^{\circ}$ bins (data points). The shaded region represents the average number of pairs expected in the case of the uniform distribution. Both the data and the uniform expectation are normalized bin-by-bin to the area of the bin, so that in the case of a uniform full-sky exposure the expectation would be flat. The overall normalization is set in such a way that the expectation in the first bin equals one.

The right panels of Figure 2 show the dependence of the $p$-value, $P(\delta)$, on the separation angle, $\delta$, for the corresponding energy. Note that $P(\delta)$ is a cumulative quantity since it takes into account all the pairs separated by angles from 0 to $\delta$. For this reason a small, but coherent over several bins, excess at angles from $10^{\circ}$ to $20^{\circ}$ on the lower left panel of Figure 2 produces a more significant feature in the corresponding $P(\delta)$ (lower right panel of Figure 2). This feature corresponds to the group of events visible on the sky map (see the lower panel of Figure 7).

When accessing the significance of departures from isotropy on the basis of $P(\delta)$ represented in Figure 2, one should take into account the fact that the angular scale of the excess is not known in advance. Thus, there is a statistical penalty for 
choosing this scale a posteriori (see Tinyakov \& Tkachev 2004 for a detailed discussion). Taking this penalty into account, none of the three examined data sets shows a significant deviation from an isotropic distribution.

Interestingly, although close clusters in the high-energy TA event set are absent, one of the TA events falls within 1.7 of a high-energy event observed by the Auger Observatory (Abreu et al. 2010). Both events have $E>10^{20} \mathrm{eV}$. The center of the doublet has the Galactic coordinates $l=36^{\circ}, b=-4.3$.

\section{CORRELATION WITH ACTIVE GALACTIC NUCLEI}

The Auger collaboration has reported a correlation (Abraham et al. 2007, 2008a) between UHECRs with $E>57 \mathrm{EeV}$ and the nearby (redshift $z \leqslant 0.018$ or, equivalently, distance $d<75 \mathrm{Mpc}$ ) AGNs from the Veron-Cetty \& Veron (VCV) catalog (Veron-Cetty \& Veron 2006). The greatest correlation was observed at the angle of 3.1 . In the control data set, the number of correlating events was 9 out of 13, which corresponds to about $69 \%$ of events. The Auger collaboration has recently updated the analysis and found that a smaller fraction of the UHECR events correlates with the same set of AGNs in the latest UHECR data set (Abreu et al. 2010) than in the original one. Out of 55 events with $E>55 \mathrm{EeV}, 21$ were found to correlate with AGNs, which corresponds to a fraction of correlating events equal to $38 \%$. In this section, we test the TA data for correlations with AGNs.

The set of 472 nearby AGNs used by Abraham et al. (2007) contains 7 objects listed at zero redshift, all in the field of view of TA. Of these seven objects, two are stars, one is a quasar with unknown redshift, one is a Seyfert 2 galaxy, two are spiral galaxies (including the Andromeda galaxy), and one is a dwarf spheroidal galaxy. We exclude these objects from the analysis, which leaves 465 objects in the AGN catalog.

The TA exposure is peaked in the Northern hemisphere, so that the AGNs visible to TA are largely different from those visible to Auger, though there is some overlap. The distribution of nearby AGNs over the sky is not uniform because of the LSS (see Section 5 for more detail) and because the VCV catalog is not complete: due to observational bias it tends to contain more objects in the Northern hemisphere. For this reason, a larger fraction of events is expected to correlate with AGNs in the TA data under the assumption that AGNs are sources of the observed UHECRs. Taking into account the distribution of nearby AGNs over the sky and assuming equal AGN luminosities in UHECR, we estimated that the correlating fraction will be $\sim 73 \%$ for TA on the basis of the original PAO claim and $\sim 43 \%$ on the basis of the updated analysis by PAO.

The sky map of TA events with $E>57 \mathrm{EeV}$ and nearby AGNs from the VCV catalog is represented in Figure 3 in Galactic coordinates. The cosmic rays are shown by filled red (correlating events) and empty blue circles (non-correlating events). AGNs are shown by black dots.

Figure 4 shows the number of TA events correlating with AGNs as a function of the total number of events with $E>$ $57 \mathrm{EeV}$ ordered according to arrival time. The black dashed line represents the expected number of random coincidences in the case of a uniform distribution calculated via MC simulation. The blue line shows the expected number of correlating events as derived from the original PAO claim. Shaded regions represent $68 \%$ and $95 \%$ CL deviations from this expectation calculated by the maximum likelihood method of Gorbunov et al. (2006). As is seen from Figure 4, present TA data are compatible with both isotropic distribution and the AGN hypothesis.

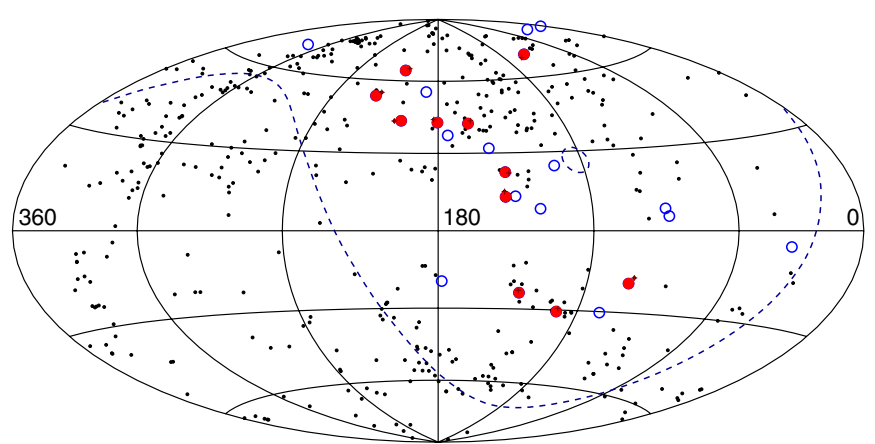

Figure 3. Hammer projection of the TA cosmic-ray events with $E>57 \mathrm{EeV}$ and nearby AGNs in the Galactic coordinates. Correlating and non-correlating events are shown by filled red and empty blue circles, respectively. AGNs are represented by black dots. The dashed line shows the boundary of the TA exposure.

(A color version of this figure is available in the online journal.)

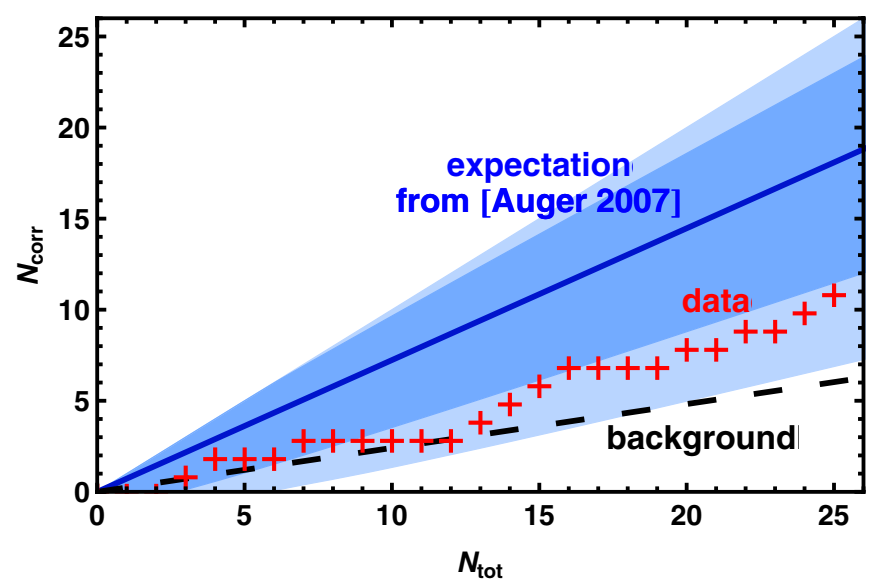

Figure 4. Number of TA events with $E>57 \mathrm{EeV}$ correlating with VCV AGNs as a function of the total number of events. The expectation according to the original PAO claim is represented by the blue line together with the $1 \sigma$ and $2 \sigma$ significance bands. The black dashed line shows the expected number of random coincidences.

(A color version of this figure is available in the online journal.)

In the full TA SD data set, there are 11 correlating events out of 25 total, while the expected number of random coincidences for this total number of events is 5.9. Making use of the binomial distribution with the probability of a single event to correlate $p_{\text {iso }}=0.24$, one finds that such an excess has a probability of $\sim 2 \%$ of occuring by chance with isotropic distribution of arrival directions.

\section{CORRELATION WITH LSS}

Even though the sources of UHECRs are not known, their distribution in space at large scales must follow that of the ordinary matter. The latter is anisotropic at scales below $\sim 100 \mathrm{Mpc}$ forming the LSS of the universe that consists of galaxy clusters, filaments, and voids. If UHECRs are not strongly deflected on their way to Earth, their distribution over the sky should correlate with the nearby structures, with overdensities corresponding to close clusters and underdensities corresponding to voids.

The amplitude of anisotropy depends on the UHECR propagation length (the larger is the propagation length, the smaller contributions of the local structures and, therefore, the anisotropy) and on the UHECR deflections. In this section, the propagation of UHECR is calculated assuming they are protons. However, it should be noted that regardless of whether 
the UHECR composition is heavy or light, their propagation length changes with energy roughly in the same way and becomes of the order of several tens of megaparsecs as the energy approaches $10^{20} \mathrm{eV}$. Thus, the most important parameter that determines the amplitude of the anisotropy at a given energy is the typical deflection angle which we denote as $\theta$ (which is, of course, very different for heavy and light composition).

The goal of this analysis is to determine which values of $\theta$ are compatible with the space distribution of the TA events. In principle, this can be done at all energies. To minimize statistical penalties, we limit our analysis to the energy thresholds of $10 \mathrm{EeV}, 40 \mathrm{EeV}$, and $57 \mathrm{EeV}$.

\subsection{Statistical Method}

To test the compatibility between the observed UHECR distribution over the sky and that expected under the LSS hypothesis (that is, the hypothesis that UHECR sources trace matter distribution in the universe), we employ the method developed by Koers \& Tinyakov (2009b) and used previously in the analysis of the HiRes data (Abbasi et al. 2010a). In this method, one first computes the UHECR flux distribution expected under the LSS hypothesis and then compares it to the observed one by the flux sampling test.

The matter distribution in the nearby universe may be inferred from the complete galaxy catalogs containing the redshift information. In this work, we use the 2MASS Galaxy Redshift Cata$\log (\mathrm{XSC} z)^{30}$ that is derived from the 2MASS Extended Source Catalog (XSC), with redshifts that have either been spectroscopically measured (for most of the objects) or derived from the Two Micron All Sky Survey (2MASS) photometric measurements. This catalog provides the most accurate information about three-dimensional galaxy distribution to date.

For the flux calculations, we use the flux-limited subsample of galaxies with apparent magnitude $m \leqslant 12.5$. For fainter objects, the completeness of the catalog degrades progressively, while their inclusion does not change the results considerably. We exclude objects closer than $5 \mathrm{Mpc}$ in order to avoid breaking the statistical description (if such objects are assumed to be sources of UHECR, they have to be treated individually). We also cut out galaxies at distances further than $250 \mathrm{Mpc}$ replacing their combined contribution by a uniform flux normalized in such a way that it provides the correct fraction of events as calculated in the approximation of a uniform source distribution. The quantitative justification of these procedures can be found in Koers \& Tinyakov (2009a). The resulting catalog contains 106,218 galaxies, which is sufficient to accurately describe the flux distribution at angular scales down to $\sim 2^{\circ}$. The UHECR flux distribution is reconstructed from this flux-limited catalog by the weighting method proposed by Lynden-Bell (1971) and adapted to flux calculations by Koers \& Tinyakov (2009a).

The XSC $z$ catalog loses completeness in the band of roughly $\pm 10^{\circ}$ around the Galactic plane and especially around the Galactic center. The size of this region is not much larger than a typical deflection of a proton even at $57 \mathrm{EeV}$, so this gap may be bridged without loss of accuracy. Away from the Galactic center at $|l|>60^{\circ}$ where only a fraction of the galaxies (the dimmer part) is missing in the catalog, we apply an $l$ - and a $b$-dependent weight correction to the remaining galaxies so as to compensate for the missing ones. In the region close to the Galactic center, $|l|<60^{\circ}$, we extrapolate the flux density from

\footnotetext{
${ }^{30}$ We are grateful to T. Jarrett for providing us with the preliminary version of
} this catalog.

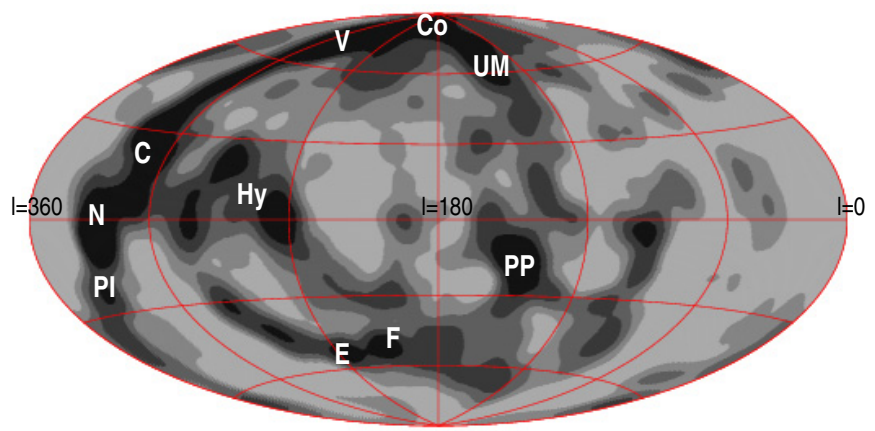

Figure 5. Sky map of expected flux at $E>57 \mathrm{EeV}$ (Galactic coordinates). The smearing angle is $6^{\circ}$. The letters indicate the nearby structures as follows: C: Centaurus supercluster $(60 \mathrm{Mpc})$; Co: Coma cluster $(90 \mathrm{Mpc})$; E: Eridanus cluster (30 Mpc); F: Fornax cluster (20 Mpc); Hy: Hydra supercluster (50 Mpc); $\mathrm{N}$ : Norma supercluster (65 Mpc); PI: Pavo-Indus supercluster (70 Mpc); PP: Perseus-Pisces supercluster (70 Mpc); UM: Ursa Major (20 Mpc); and V: Virgo cluster $(20 \mathrm{Mpc})$.

(A color version of this figure is available in the online journal.)

the adjacent regions in a straightforward manner. The latter is not an accurate procedure; however, the Galactic center region overlaps with the TA exposure only slightly, and this inaccuracy is not important for our results as can be checked by excluding this region from the analysis.

When propagating the UHECR primary particles from a source to the Earth, we assume them to be protons and take full account of the attenuation processes. The injection index at the source is taken to be 2.4 , which is compatible with the UHECR spectrum observed by HiRes and TA (Abu-Zayyad et al. 2012a) assuming proton composition and the source evolution parameter $m=4$ (Gelmini et al. 2007). We also assume that the effects of both the Galactic and extragalactic magnetic fields can be approximated by a single parameter, the Gaussian smearing angle $\theta$. We consider $\theta$ a free parameter and vary it in the range $2^{\circ}-20^{\circ}$. In general, the deflections of UHECR in magnetic fields contain both random and regular parts, the latter being due to the regular component of the GMF. The regular deflections are not Gaussian. However, the statistical test we use here is not sensitive to the coherent character of deflections provided they do not exceed $10^{\circ}-20^{\circ}$ as set by the typical size of the flux variations due to local structures (cf. Figure 5). Thus, for most of the analysis we will use the Gaussian smearing to represent all the deflections without making the distinction between the regular and random ones. Later, in Section 5.4 in the case of the lowest energy set and the largest deflections, we will discuss the effect of explicitly accounting for the regular component of the GMF.

To calculate the expected flux, we assume that UHECR sources follow the space distribution of galaxies. The simplest way to realize this assumption in practice is to assign each galaxy an equal luminosity in UHECRs. This is a good approximation if the density of the UHECR sources is sufficiently high (so that many sources are present in local structures contributing to the anisotropy). The contribution of each galaxy to the total flux is then calculated taking into account the distance of the source and the corresponding flux attenuation. Individual contributions are smeared with the Gaussian width $\theta$, so that the flux at a given point of the sky is a sum of contributions of all the galaxies within the angular distance of order $\theta$. Further details on the flux calculation can be found in Koers \& Tinyakov (2009b), Koers \& Tinyakov (2009a), and Abbasi et al. (2010a). 
Figure 5 shows the flux map calculated by the above procedure for an energy threshold of $57 \mathrm{EeV}$ and smearing angle $\theta=6^{\circ}$, not yet modulated with the TA exposure. The darker regions correspond to higher flux. A band of each color integrates to one-fifth of the total flux. One can identify the nearby structures that are marked by letters on the plot as explained in the caption.

The next step is to compare the calculated flux distribution to the actual distribution of the TA events and determine whether they are statistically compatible. In this work, we use the flux sampling test proposed by Koers \& Tinyakov (2009b). The starting point is the map of the expected flux calculated as explained above. One reads off the flux values at positions of the data events. This gives a set of numbers which we refer to as the "data set." One may say that the cosmic-ray events sample the flux map in a particular way that depends on their space distribution. One then generates a large number of $\mathrm{MC}$ events which are distributed according to the expected flux and reads off the flux values at their positions. This gives the set of flux values which we refer to as the "MC set." If the angular distribution of the data and $\mathrm{MC}$ events is the same, so must be the distributions of the flux values in the data and MC sets. These two distributions may be compared by the parameter-free K-S test.

The result of the K-S test is the $p$-value which shows whether or not the data and MC flux sets are drawn from the same parent distribution. If this $p$-value is low, the two distributions of flux values are different and, therefore, the angular distributions of data and MC sets are different.

\subsection{Estimate of Statistical Power of the Flux Sampling Test}

An important characteristic of a statistical test is its ability to discriminate between two hypotheses or the statistical power. For the case at hand, the statistical power is the probability to rule out the LSS hypothesis at $95 \% \mathrm{CL}$ if the cosmic-ray distribution is isotropic. The closer the statistical power is to one, the more sensitive the test. The statistical power provides an a priori idea of what kind of sensitivity can be reached with the given number of events.

In general, the statistical power increases with smaller smearing angles since this improves the contrast in the flux map. For the same reason, the statistical power increases with energy (the UHECR propagation length becomes shorter and the relative contribution of the local structures is therefore enhanced). Also, the statistical power increases with the number of events.

We have calculated the statistical power of the flux sampling test in case of TA for the three energy thresholds of $10 \mathrm{EeV}$, $40 \mathrm{EeV}$, and $57 \mathrm{EeV}$, and smearing angles varying from $2^{\circ}$ to $14^{\circ}$. We have found that for the actual number of events in the TA data set, the statistical power is below $50 \%$ for smearing angles $\theta>9^{\circ}, \theta>3^{\circ}$, and $\theta>4^{\circ}$ for the above three energy thresholds, respectively.

The case $E>57 \mathrm{EeV}$ is shown in Figure 6. The various curves in the plot correspond to different number of events (note that the actual number of events in the TA data set with $E>57 \mathrm{EeV}$ is 25$)$. The gray region represents the expected range of deflections in the GMF in the case of protons.

\subsection{Results}

First, we check the compatibility of the TA event sets with the isotropic distribution. To this end, we generate an isotropic flux map modulated with the TA exposure. This map is independent

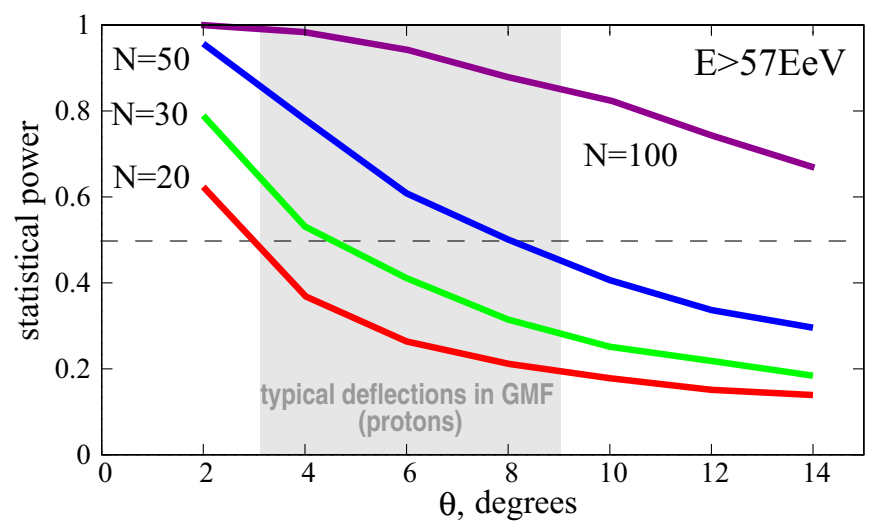

Figure 6. Statistical power of the flux sampling test at $E>57 \mathrm{EeV}$ as a function of the smearing angle. Different curves correspond to different number of events, as indicated on the plot. The actual number of events in the TA data set with $E>57 \mathrm{EeV}$ is 25 . The gray region shows the expected range of deflections in the Galactic magnetic field in the case of protons.

(A color version of this figure is available in the online journal.)

of energy and smearing angle. We then test the compatibility of the TA event set for $E>10 \mathrm{EeV}, E>40 \mathrm{EeV}$, and $E>57 \mathrm{EeV}$ with this map. The flux sampling test gives the $p$-values $0.5,0.9$, and 0.6 for the three data sets, respectively. Thus, at all three energy thresholds the data appear to be compatible with an isotropic distribution.

Next, we examine the compatibility of the TA event sets with the LSS hypothesis. Figure 7 shows the sky maps of the expected flux at energy thresholds of $10 \mathrm{EeV}, 40 \mathrm{EeV}$, and $57 \mathrm{EeV}$ (top to bottom) and the smearing angle of $6^{\circ}$. The white dots represent the arrival directions of the TA events. The bands are drawn in the same way as in Figure 5, i.e., each band integrates to onefifth of the total flux. This means, in particular, that if the LSS model were true each band would contain one-fifth of the total number of events in average. Note that the configuration of the bands changes with energy because of the energy dependence of the propagation length.

The results of the flux sampling tests are presented in Figure 8. The $p$-values are shown as a function of the smearing angle at energy thresholds of $10 \mathrm{EeV}, 40 \mathrm{EeV}$, and $57 \mathrm{EeV}$. Each point represents the $p$-value obtained by the flux sampling test at the corresponding energy threshold and smearing angle.

As one can see from the plots, for $E>40 \mathrm{EeV}$ and $E>57 \mathrm{EeV}$ the data are compatible with the structure hypothesis at the $95 \%$ C.L. The decrease of the $p$-values slightly below the $95 \%$ C.L. in the case $E>57 \mathrm{EeV}$ cannot be assigned a real significance in view of the penalty factors for trials (e.g., three energy thresholds).

Although large smearing angles do not have a straightforward physical interpretation in view of the Gaussian approximation used, we have investigated the behavior of the $p$-values corresponding to the case $E>57 \mathrm{EeV}$ for larger smearing angles and found that it fluctuates around $p \simeq 0.05$ for angles as large as $\theta \sim 50^{\circ}$ and then goes to $p \simeq 1$. Such behavior may arise because the flux map for $E>57 \mathrm{EeV}$ remains anisotropic even for very large smearing angles.

At the energy threshold of $E>10 \mathrm{EeV}$ the situation is somewhat different. The data are incompatible with the structure model up to angles of order $20^{\circ}$. In view of the large deflections in magnetic fields at low energies, such behavior is expected. One should be careful, however, with the interpretation of this result. First, Figure 8 does not include the penalty for the 


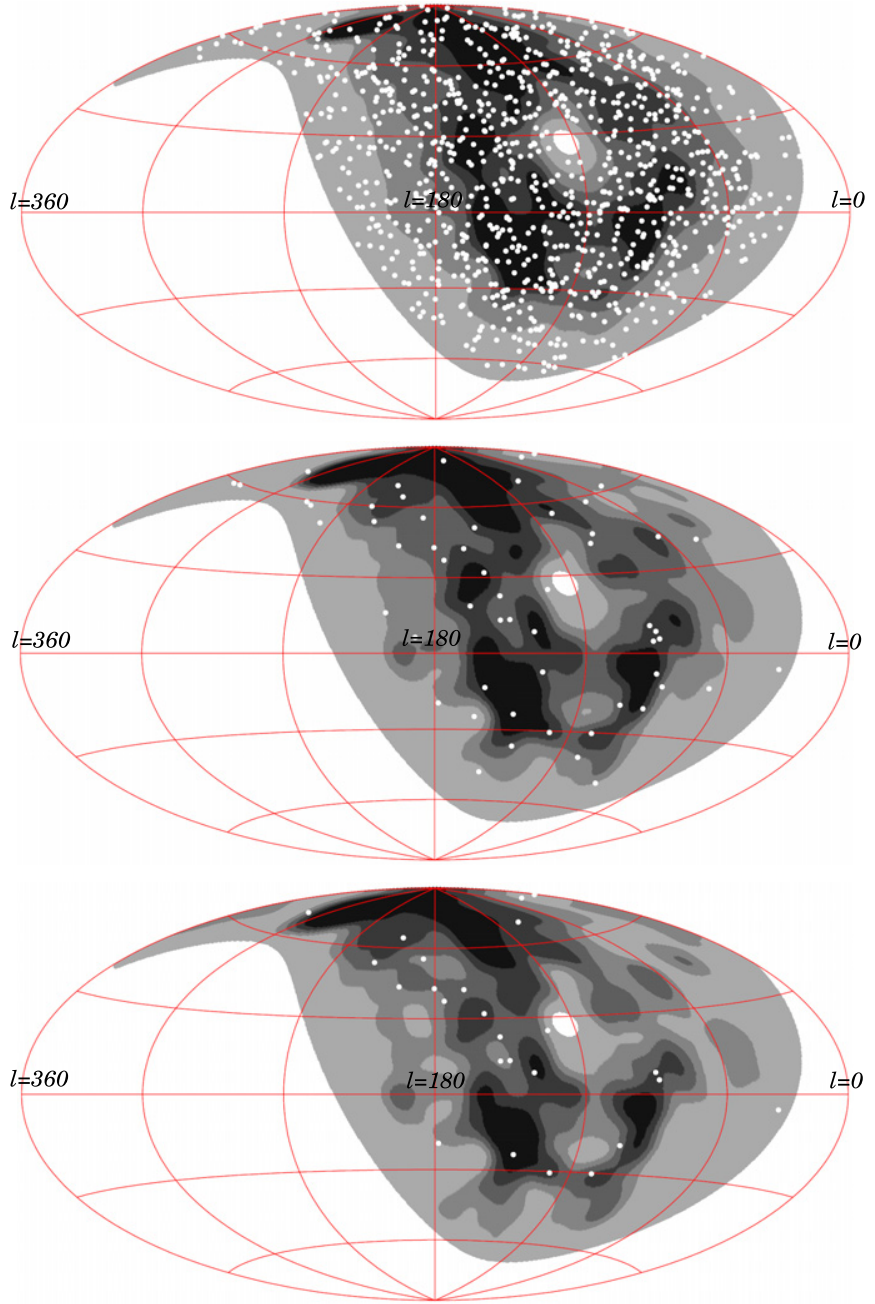

Figure 7. Sky maps of the expected flux at energy thresholds of $10 \mathrm{EeV}, 40 \mathrm{EeV}$, and $57 \mathrm{EeV}$ (from top to bottom) in Galactic coordinates with the TA events superimposed (white dots). The smearing angle is $6^{\circ}$.

(A color version of this figure is available in the online journal.)

number of trials. Second, at $E>10 \mathrm{EeV}$ the uncertainties in the flux calculation due to the choice of the model parameters (in particular, the injection index and the evolution parameter) are the largest. Finally, if the smearing angle is attributed to deflections in the magnetic fields, the dominant contribution is likely to come from the regular component of the GMF, as discussed in the next section. Such large and regular deflections require a more accurate modeling, which we attempt in the next section.

\subsection{Accounting for the Galactic Magnetic Field}

The deviation from the structure model at $E>10 \mathrm{EeV}$ and small smearing angles is an indication that magnetic field deflections play an important role in the distribution of the UHECR arrival directions. In general, several contributions to the deflections are expected. First, there are deflections produced by intergalactic magnetic fields. These fields are known quite poorly. They are usually thought to obey the upper bound of $B \lesssim 10^{-9}$ $\mathrm{G}$ with a correlation length, $l \lesssim 1 \mathrm{Mpc}$ (Kronberg 1994). With these parameters, a proton of energy $10 \mathrm{EeV}$ coming from 50 Mpc would be deflected by $\sim 20^{\circ}$. However, there are indications that the extragalactic magnetic fields may be several orders of magnitude smaller (Dolag et al. 2005) than the upper bound.
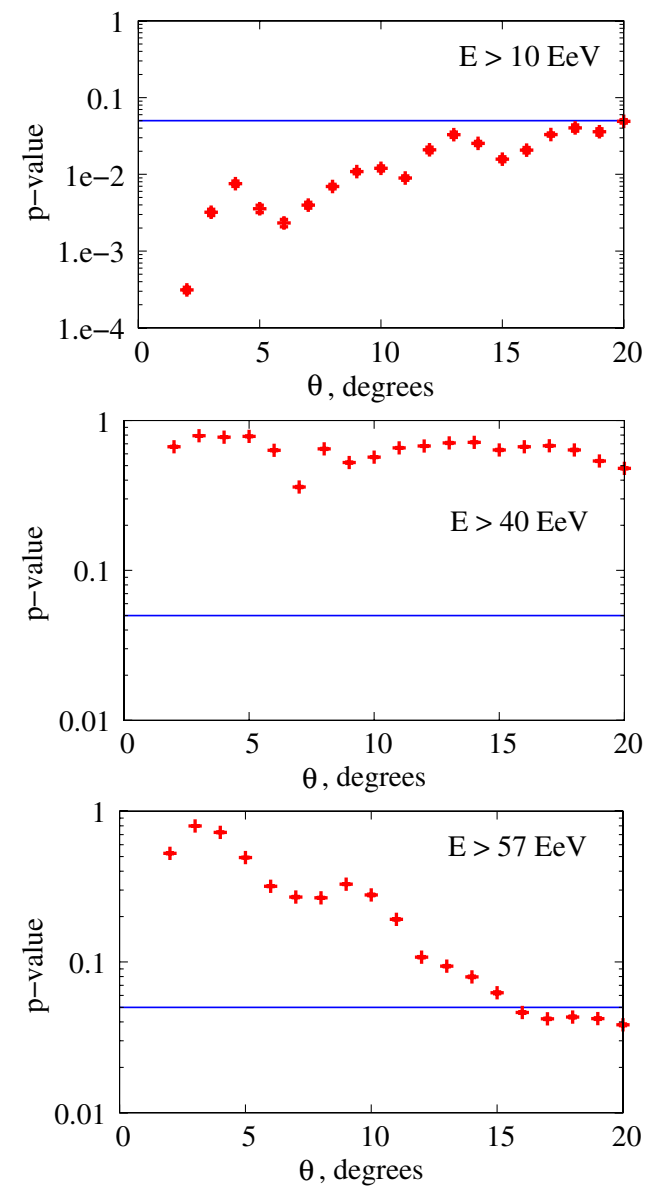

Figure 8. Results of the statistical test for the compatibility between the data and the LSS hypothesis. The $p$-values (red points) are shown as a function of the smearing angle $\theta$. Low $p$-values indicate incompatibility with the LSS model. The horizontal line shows a confidence level of $95 \%$. The three panels correspond to energy thresholds of $10 \mathrm{EeV}, 40 \mathrm{EeV}$, and $57 \mathrm{EeV}$ from top to bottom, as indicated on the plots.

(A color version of this figure is available in the online journal.)

Second, UHECRs are deflected in the regular component of the GMF. The regular GMF is known much better than extragalactic fields. It can be inferred, e.g., from the Faraday rotation measures of Galactic and extragalactic radio sources. According to recent studies, a typical deflection of a $10 \mathrm{EeV}$ proton would be $20^{\circ}-40^{\circ}$ (Pshirkov et al. 2011). This is comparable or larger than the deflection in the extragalactic field.

Finally, the Galactic field has a random component. Although the amplitude of this component is a few times larger than the regular one, its contribution into the deflections is subdominant (or at most comparable) to that of the regular component (Tinyakov \& Tkachev 2005) due to its random character.

From this discussion it is clear that the regular part of the magnetic field most likely provides the dominant contribution into the UHECR deflections. At low energies when the magnitude of the deflections becomes large, Gaussian smearing is not a good approximation for such deflections. They have to be taken into account explicitly.

In order to see whether or not the deflections in the regular GMF can be a reason for the discrepancy between the data and the LSS model we have repeated the analysis of Section 5.3 including the regular GMF. The presence of the regular magnetic field is taken into account by modifying the expected flux 

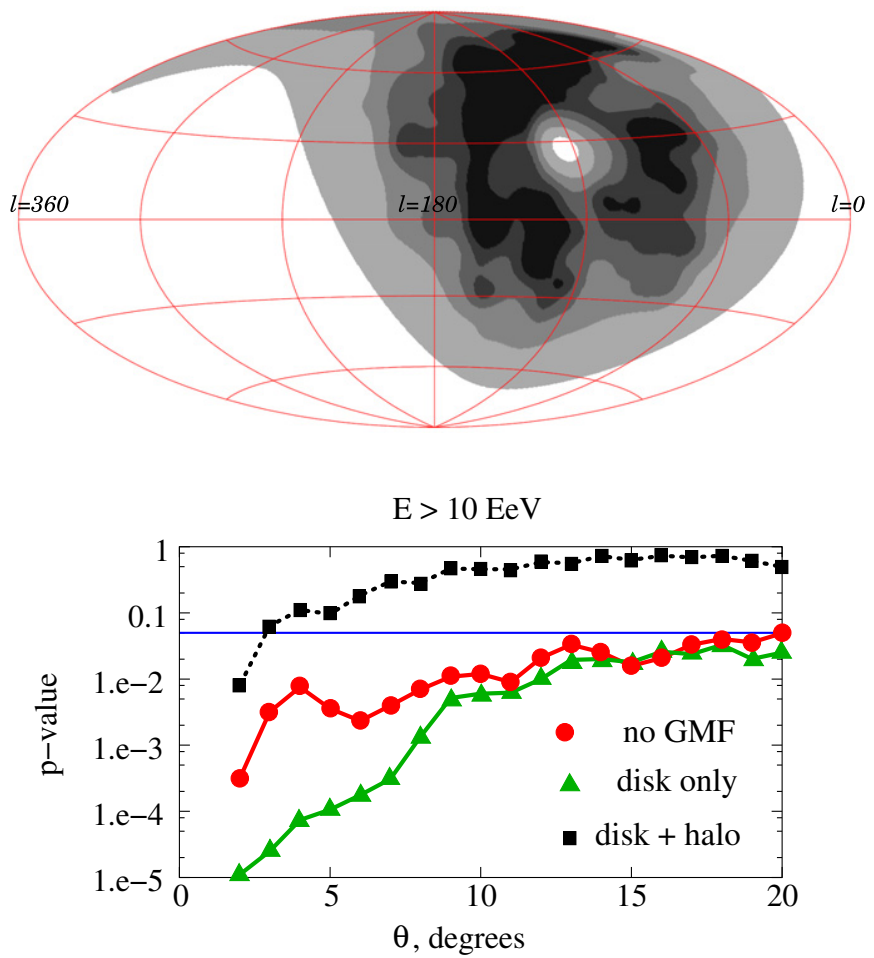

Figure 9. Upper panel: the sky map of the expected flux for $E>10 \mathrm{EeV}$ and smearing angle $6^{\circ}$ taking into account the GMF (Galactic coordinates). The parameters of GMF are as follows: the magnitude of the halo is $4 \mu \mathrm{G}$ and the thickness of the halo is $1.5 \mathrm{kpc}$. Note the absence of overdensity in the direction of the Virgo cluster. Lower panel: the result of the statistical test of the compatibility between the TA event set with $E>10 \mathrm{EeV}$ and the LSS hypothesis for different models of GMF: no magnetic field (circles), disk component only (triangles), both disk and halo components (squares). The horizontal line shows the confidence level of $95 \%$. Low $p$-values indicate incompatibility.

(A color version of this figure is available in the online journal.)

distribution. The smearing angle remains a free parameter; it accounts for random deflections in the extragalactic fields and in the random component of the GMF. The statistical test itself remains unchanged.

We adopt the recent GMF model by Pshirkov et al. (2011). This model has been obtained by fitting the GMF model parameters to the latest catalog of the Faraday rotation measures of extragalactic sources. In addition to the disk field, this model also contains a toroidal halo field.

Although the fits to the Faraday rotation measures constrain the parameters of the GMF, some combinations of these parameters are constrained rather poorly. In particular, the magnitude of the halo field is degenerate with the halo height above the Galactic disk: making the halo field stronger and simultaneously higher above the disk does not strongly affect the rotation measures. Thus, some freedom remains in the choice of the GMF parameters. The question is whether or not this freedom can be used to bring the arrival directions of UHECR into accord with the LSS hypothesis without contradicting the Faraday rotation data.

We have found that the compatibility with the LSS model cannot be reached without the halo field. When the halo is included, the compatibility with the LSS model is possible, although the required halo field is rather strong (but still compatible with the data on the Faraday rotation measures).

An example of the flux map with the GMF included is shown in the upper panel of Figure 9. The flux distribution is calculated for the case $E>10 \mathrm{EeV}$ and smearing angle of $6^{\circ}$. The magnetic field parameters are as follows: the magnitude of the halo is 4 $\mu \mathrm{G}$ and the thickness of the halo is $1.5 \mathrm{kpc}$. Note that after the inclusion of GMF the Virgo region has moved away from the TA field of view, and the expected flux distribution has become closer to the uniform one.

The results of the flux sampling test of the LSS model with the regular GMF included are shown in the lower panel of Figure 9. The black squares represent the $p$-values in the case of the GMF with the parameters described above. For comparison, the red circles show the $p$-values in the absence of GMF (the same as the upper panel of Figure 8), while the green triangles represent the case of GMF with the disk component only. One can see that the regular GMF can produce deflections that make the data for $E>10 \mathrm{EeV}$ compatible with the LSS model for all but the smallest smearing angles. Thus, the discrepancy between the LSS hypothesis and the TA data with $E>10 \mathrm{EeV}$ can, in principle, be explained by the deflections in the regular GMF.

\section{SUMMARY AND CONCLUSIONS}

In this paper, we present a search for anisotropy in the TA data collected over the period of about 40 months, which is the largest UHECR data set to date in the Northern hemisphere. The main focus of this paper is on checking the existing claims: smallscale clustering, correlation with nearby AGNs, and correlation with the LSS.

The results are summarized as follows.

1. The TA data show no clustering of the UHECR events at small scales, neither at the angular scale of 2.5 in the set with $E>40 \mathrm{EeV}$ as reported by the AGASA experiment nor at any angular scale from $0^{\circ}$ to $40^{\circ}$ in the data sets with $E>10 \mathrm{EeV}, E>40 \mathrm{EeV}$, and $E>57 \mathrm{EeV}$. There is a hint of grouping of events at angular scales of $20^{\circ}-30^{\circ}$ at the highest energies; however, the statistical significance of this feature is insufficient for a definite conclusion.

2. There is no statistically significant correlation of the TA data with $E>57 \mathrm{EeV}$ with the positions of nearby AGNs from the VCV catalog using the parameters reported by the PAO (angular scale of 3.1 and redshift cut in the VCV catalog $z \leqslant 0.018$ ). Out of 25 observed events with $E>57 \mathrm{EeV}$, 11 have been found to correlate with positions of nearby AGNs, while 5.9 are expected on average from random coincidences (chance probability of $2 \%$ ).

3. The TA event sets with $E>10 \mathrm{EeV}, E>40 \mathrm{EeV}$, and $E>57 \mathrm{EeV}$ appear compatible with a uniform distribution according to the flux sampling test. The sets with $E>40 \mathrm{EeV}$ and $E>57 \mathrm{EeV}$ are also compatible, at $95 \% \mathrm{CL}$, with a model which assumes that sources follow the LSS of the universe (LSS model). The set with $E>10 \mathrm{EeV}$ is not compatible, at $95 \% \mathrm{CL}$, with the LSS model unless the deflections of these UHECRs exceed $20^{\circ}$.

4. The set with $E>10 \mathrm{EeV}$ can be made compatible with the LSS model, at smearing angles larger than $\sim 3^{\circ}$, by including the effect of the regular component of the GMF and assuming a realistic model for the latter. The smearing angle in this case represents the deflections in the random Galactic and extragalactic fields.

From the analysis presented, one concludes that there is no apparent deviation from isotropy in the present TA data. At high energies, this may be merely due to an insufficient number of events. However, if this tendency persists at several times larger statistics, it will be difficult to reconcile with the proton composition of UHECR regardless of the source nature: if the 
Table 1

List of Telescope Array Events with $E \geqslant 57 \mathrm{EeV}$ and Zenith Angle $\theta<45^{\circ}$ Recorded from 2008 May 11 to 2011 September 15

\begin{tabular}{lrrrr}
\hline \hline Date and Time $(\mathrm{UTC})$ & $\theta(\mathrm{deg})$ & $E(\mathrm{EeV})$ & $l(\mathrm{deg})$ & $b(\mathrm{deg})$ \\
\hline 2008 Jun 25 19:45:52 & 32.8 & 82.6 & 178.6 & -19.4 \\
2008 Jul 15 05:26:31 & 34.4 & 57.7 & 90.5 & 8.0 \\
2008 Aug 10 12:45:04 & 38.0 & 122.6 & 102.7 & -19.2 \\
2008 Nov 8 14:30:41 & 15.5 & 60.0 & 198.0 & 43.1 \\
2008 Dec 30 10:49:32 & 4.5 & 59.7 & 187.0 & 55.3 \\
2009 Jan 22 22:54:22 & 31.3 & 58.0 & 89.3 & 5.2 \\
2009 Mar 28 04:36:08 & 34.2 & 81.2 & 152.8 & 22.5 \\
2009 Mar 29 03:43:34 & 20.7 & 75.0 & 158.1 & 31.9 \\
2009 May 19 02:19:52 & 42.5 & 64.6 & 25.8 & 77.3 \\
2009 Sep 19 08:45:52 & 34.7 & 62.0 & 140.5 & 8.4 \\
2010 Jan 8 07:17:31 & 19.5 & 57.5 & 175.6 & 37.2 \\
2010 Jan 21 03:53:51 & 23.4 & 61.2 & 149.8 & 13.1 \\
2010 Feb 22 07:10:34 & 14.5 & 63.5 & 165.7 & 42.0 \\
2010 Aug 29 21:20:45 & 36.5 & 69.9 & 180.3 & 42.4 \\
2010 Aug 30 20:50:45 & 20.0 & 93.3 & 98.3 & 69.7 \\
2010 Sep 19 07:05:00 & 23.6 & 66.8 & 129.1 & -30.6 \\
2010 Sep 21 20:37:06 & 21.1 & 163.0 & 2.8 & 76.0 \\
2011 Jan 5 00:56:23 & 9.3 & 67.4 & 110.0 & -30.4 \\
2011 Feb 28 16:16:26 & 39.3 & 137.6 & 35.5 & -5.0 \\
2011 Apr 17 20:20:29 & 34.2 & 74.7 & 153.7 & 12.9 \\
2011 Jul 13 19:12:34 & 42.6 & 65.6 & 132.1 & 24.7 \\
2011 Jul 22 22:15:41 & 11.6 & 62.2 & 204.5 & 64.6 \\
2011 Jul 24 23:17:22 & 36.3 & 61.8 & 316.5 & 69.5 \\
2011 Jul 28 15:21:08 & 19.6 & 89.0 & 147.0 & -23.7 \\
2011 Aug 28 21:14:19 & 31.6 & 63.3 & 215.6 & 53.2 \\
\hline
\end{tabular}

sources within the GZK volume are numerous, they must follow the (inhomogeneous) matter distribution. If the source density is small so that there are only a few within the GZK volume, this very fact will produce anisotropy.

At lower energies the deflections are expected to be large even for protons, which makes the distribution of the events more isotropic. However, the number of events is much larger as well, so that even small deviations from isotropy may become detectable as the statistics increases. The fact that for $E>10 \mathrm{EeV}$ the distribution of the events is not compatible with the LSS model without assuming a large $\left(\gtrsim 20^{\circ}\right)$ smearing angle may indicate that we are observing the first manifestation of UHECR deflections in the GMFs. The possibility to reconcile the observed UHECR distribution with the LSS model by correcting for the deflections in the realistic model of GMF is in accord with this interpretation.

The Telescope Array experiment is supported by the Japan Society for the Promotion of Science through Grants-inAid for Scientific Research on Specially Promoted Research (21000002) "Extreme Phenomena in the Universe Explored by Highest Energy Cosmic Rays" and for Scientific Research (S) (19104006), and the Inter-University Research Program of the Institute for Cosmic Ray Research; by the U.S. National Science Foundation awards PHY-0307098, PHY-0601915, PHY0703893, PHY-0758342, PHY-0848320, PHY-1069280, and PHY-1069286 (Utah) and PHY-0649681 (Rutgers); by the National Research Foundation of Korea (2006-0050031, 20070056005, 2007-0093860, 2010-0011378, 2010-0028071, 20110002617, R32-10130); by the Russian Academy of Sciences, RFBR grants 10-02-01406a and 11-02-01528a (INR), IISN project No. 4.4509.10 and Belgian Science Policy under IUAP VI/11 (ULB); by the Grant-in-Aid for the Scientific Research (S) No. 19104006 by the Japan Society for the Promotion of
Science. The foundations of Dr. Ezekiel R. and Edna Wattis Dumke, Willard L. Eccles, and the George S. and Dolores Dore Eccles all helped with generous donations. The State of Utah supported the project through its Economic Development Board, and the University of Utah through the Office of the Vice President for Research. The experimental site became available through the cooperation of the Utah School and Institutional Trust Lands Administration (SITLA), the U.S. Bureau of Land Management, and the U.S. Air Force. We also thank the people and the officials of Millard County, Utah, for their steadfast and warm support. We gratefully acknowledge the contributions from the technical staffs of our home institutions and the University of Utah Center for High Performance Computing (CHPC).

\section{APPENDIX \\ LIST OF EVENTS WITH $E>57 \mathrm{EeV}$}

In this Appendix, we present the list of events with energy $E>57 \mathrm{EeV}$ and zenith angle $\theta<45^{\circ}$ that have been recorded by the SD of the TA from 2008 May 11 to 2011 September 15. During this period, 25 such events were observed. Table 1 shows the arrival date and time of these events, the zenith angle $\theta$, energy in units of $\mathrm{EeV}$, and Galactic coordinates $l$ and $b$ in degrees. The angular resolution of these events is $\sim 1.5$, while the energy resolution is better than $20 \%$.

\section{REFERENCES}

Abbasi, R., Abu-Zayyad, T., Allen, M., et al. 2008a, Phys. Rev. Lett., 100, 101101

Abbasi, R., Abu-Zayyad, T., Allen, M., et al. 2008b, Astropart. Phys., 30, 175

Abbasi, R., Abu-Zayyad, T., Allen, M., et al. 2010a, ApJ, 713, L64

Abbasi, R., Abu-Zayyad, T., Al-Seady, M., et al. 2010b, Phys. Rev. Lett., 104, 161101

Abbasi, R., Abu-Zayyad, T., Amann, J. F., et al. 2006, ApJ, 636, 680

Abraham, J., Abreu, P., Aglietta, M., et al. 2007, Science, 318, 938

Abraham, J., Abreu, P., Aglietta, M., et al. 2008a, Astropart. Phys., 29, 188

Abraham, J., Abreu, P., Aglietta, M., et al. 2008b, Phys. Rev. Lett., 101, 061101

Abraham, J., Abreu, P., Aglietta, M., et al. 2010, Phys. Rev. Lett., 104, 091101

Abreu, P., Aglietta, M., Ahn, E. J., et al. 2010, Astropart. Phys., 34, 314

Abu-Zayyad, T., Aida, R., Allen, M., et al. 2012a, NIMPA, 689, 87

Abu-Zayyad, T., Aida, R., Allen, M., et al. 2012b, arXiv:1201.4964

Bird, D. J., Dai, H. Y., Dawson, B. R., et al. 1999, ApJ, 511, 739

Dolag, K., Grasso, D., Springel, V., \& Tkachev, I. 2005, J. Cosmol. Astropart. Phys., JCAP01(2005)009

Dubovsky, S. L., Tinyakov, P. G., \& Tkachev, I. I. 2000, Phys. Rev. Lett., 85, 1154

Gelmini, G. B., Kalashev, O. E., \& Semikoz, D. V. 2007, J. Cosmol. Astropart. Phys., JCAP11(2007)002

Giacinti, G., Kachelriess, M., Semikoz, D., \& Sigl, G. 2010, J. Cosmol. Astropart. Phys., JCAP08(2010)036

Glushkov, A. 2001, JETP Lett., 73, 313

Glushkov, A., \& Pravdin, M. 2001, J. Exp. Theor. Phys., 92, 887

Gorbunov, D. S., Tinyakov, P. G., Tkachev, I. I., \& Troitsky, S. V. 2004, JETP Lett., 80, 145

Gorbunov, D. S., Tinyakov, P. G., Tkachev, I. I., \& Troitsky, S. V. 2006, J. Cosmol. Astropart. Phys., JCAP01(2006)025

Greisen, K. 1966, Phys. Rev. Lett., 16, 748

Han, J. L., Manchester, R. N., Lyne, A. G., Qiao, G. J., \& van Straten, W. 2006, ApJ, 642, 868

Hayashida, N., Honda, K., Honda, M., et al. 1996, Phys. Rev. Lett., 77, 1000

Kachelriess, M., \& Semikoz, D. 2005, Astropart. Phys., 23, 486

Kashti, T., \& Waxman, E. 2008, J. Cosmol. Astropart. Phys., JCAP05(2008)006

Kewley, L. J., Clay, R. W., \& Dawson, B. R. 1996, Astropart. Phys., 5, 69

Koers, H. B. J., \& Tinyakov, P. 2009a, MNRAS, 399, 1005

Koers, H. B. J., \& Tinyakov, P. 2009b, J. Cosmol. Astropart. Phys., JCAP04(2009)003

Kronberg, P. P. 1994, Rep. Prog. Phys., 57, 325

Lynden-Bell, D. 1971, MNRAS, 155, 95 
Pshirkov, M., Tinyakov, P., Kronberg, P., \& Newton-McGee, K. 2011, ApJ, 738 192

Stanev, T., Biermann, P. L., Lloyd-Evans, J., Rachen, J. P., \& Watson, A. A. 1995, Phys. Rev. Lett., 75, 3056

Sun, X. H., Reich, W., Waelkens, A., \& Enßlin, T. A. 2008, A\&A, 477, 573

Takami, H., Inoue, S., \& Yamamoto, T. 2012, J. Astropart. Phys., 35, 767

Tameda, Y. 2010, AIP. Conf. Proc., 1367, 110

Tinyakov, P. G., \& Tkachev, I. I. 2001, JETP Lett., 74, 1
Tinyakov, P. G., \& Tkachev, I. I. 2004, Phys. Rev. D, 69, 128301

Tinyakov, P. G., \& Tkachev, I. I. 2005, Astropart. Phys., 24, 32

Tokuno, H., Tameda, Y., Takeda, M., et al. 2012, Nucl. Instrum. Methods Phys. Res., 676, 54

Veron-Cetty, M.-P., \& Veron, P. 2006, A\&A, 455, 773

Yoshiguchi, H., Nagataki, S., \& Sato, K. 2004, ApJ, 614, 43

Yoshiguchi, H., Nagataki, S., Tsubaki, S., \& Sato, K. 2003, ApJ, 586, 1211

Zatsepin, G. T., \& Kuzmin, V. A. 1966, JETP Lett., 4, 78 